\title{
On the radiative lifetime of free-moving two-dimensional excitons
}

\author{
A. V. Paraskevov \\ National Research Centre "Kurchatov Institute", Kurchatov Sq. 1, Moscow 123182, Russia
}

\begin{abstract}
A simple microscopic mechanism explaining the linear dependence of the radiative lifetime of freemoving two-dimensional excitons on their effective temperature is suggested. It is shown that there exists a characteristic effective temperature (of about few Kelvin) defined by the exciton-acoustic phonon interaction at which the radiative lifetime is minimal. Below this temperature the lifetime starts to increase with decreasing temperature. The correspondence with previous theoretical and experimental results is discussed.
\end{abstract}

PACS numbers: 71.35.-y, 78.60.-b, 78.67.De

Keywords: Exciton radiative lifetime, Quantum wells, Exciton-acoustic phonon interaction

\section{INTRODUCTION}

The mechanism of optical recombination of freemoving two-dimensional (2D) excitons in semiconductor quantum wells (QWs) is an intriguing open question in the field of exciton physics, especially, in the light of recent experiments on spatially-resolved exciton luminescence indicating non-trivial diffusion of excitons in the QW plane [1]. In fact, optical decay of a free-moving $2 \mathrm{D}$ exciton leads to the formation of either a threedimensional (3D) photon or a quasi-2D exciton polariton, if the exciton system is placed in a resonant planar microcavity [2 7]. The decay can depend on interactions of excitons with acoustic phonons and defects. Indeed, the presence of a "third body" (e.g., 3D acoustic phonons) could open a fast channel of radiative decay similar to three-body recombination in plasmas and cold gases. In what follows, we study the influence of excitonacoustic phonon interaction on optical recombination of free-moving excitons in a narrow QW, without any resonant cavity.

The first phenomenological theory of optical decay of free-moving QW excitons was suggested in Ref.[8]. It was assumed that the excitons had a Maxwellian distribution over momenta with some effective temperature $T$. The influence of the exciton-phonon interaction on exciton radiative lifetime $\tau_{R}$ was taken into account by sharing the oscillator strength of an exciton with zero momentum equally among all momentum states within a phenomenological spectral width. It was found that $\tau_{R} \propto T$. This result was justified by the reasonable agreement with experimental data for the case of excitons nearly thermalized to the lattice temperature $T_{0}$, i.e., at $T \approx T_{0}$. From the physical point of view this means that the increase of $\tau_{R}$ with $T_{0}$ is due to the fact that the scattering of excitons on phonons can lead to a decrease of the fraction of excitons (in momentum space) that are able to decay radiatively.

In Refs. [3, [4, [6] the optical recombination rate $\Gamma(p)$ of an exciton with in-plane momentum $p$ was derived assuming that only the exciton with momentum $p<k=$ $\omega_{0} / c$ can decay radiatively, where $k$ and $\omega_{0}$ are wave vector and frequency of a 3D photon, respectively, and $c$ is the speed of light in the medium. The averaging of $\Gamma(p)$ over the thermal distribution of excitons gave the previous result $\tau_{R} \propto T[4,[6]$ (see Appendix). However, if one considers the case when a 2D exciton optically decays with the simultaneous emission of a 3D acoustic phonon, then the condition $p<k$ for the radiative decay is apparently invalid even at $T_{0}=0$.

In this paper we study the influence of exciton-acoustic phonon interaction on optical decay of free-moving QW excitons for the case when the exciton kinetic energy is small enough to neglect optical phonon emission. In particular, starting from the premise that free-moving $2 \mathrm{D}$ exciton decays only with the formation of a 3D photon and a 3D acoustic phonon (or, equivalently, that the exciton decays just after scattering from the phonon with some definite momentum), we have derived the dependence $\tau_{R} \propto T$ at high $T$, but in completely different way compared to Ref.[4]. In addition, our model gives an intuitively-expected upturn of the dependence at very low $T$. Note that the above premise is based on two general reasons: (i) an exciton is a metastable system so any additional interaction would likely facilitate its decay; (ii) the exciton-acoustic phonon interaction is something unavoidable even in the case of zero lattice temperature, where excitons can only emit phonons.

\section{MODEL}

Let us consider a quasi-equilibrium system of freemoving 2D excitons with effective temperature $T$, where excitons can interact with 3D longitudinal acoustic (LA) phonons. Then exciton distribution function $f_{p}$ over quasi-momentum $\mathbf{p}$ is Maxwellian, $f_{p}=$ $\exp \left(-\left(E_{p}-\mu\right) / T\right)$, where dispersion law $E_{p}=p^{2} / 2 m$, $m$ is the exciton effective mass, and $\mu<0$ corresponds to the chemical potential of the exciton system, $|\mu| \gg T$. The exciton density is $n=\frac{1}{S} \sum_{\mathbf{p}} f_{p}=$ $m T \exp (\mu / T) /\left(2 \pi \hbar^{2}\right)$, where $S$ is the $\mathrm{X}$-Y plane area. For clarity we set lattice temperature $T_{0}=0$, so the excitons can only emit phonons. In what follows we use the notation: $\mathbf{p}$ is $2 \mathrm{D}$ exciton momentum in $\mathrm{X}-\mathrm{Y}$ plane, $\mathbf{k}$ is 

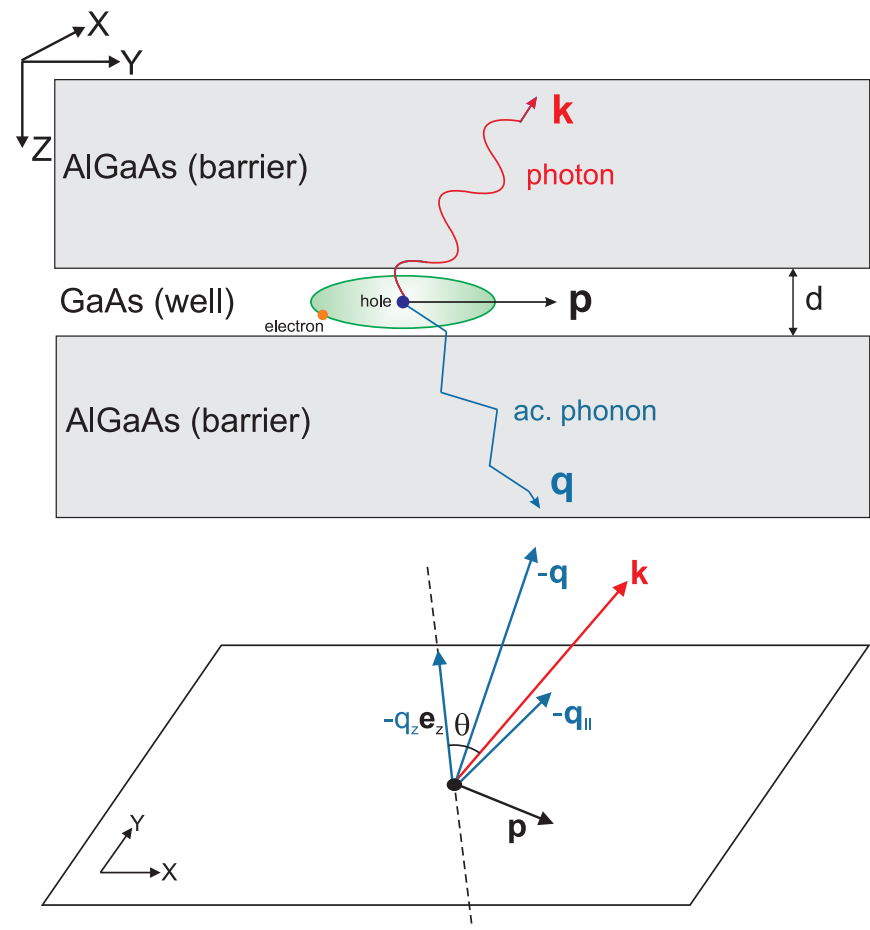

Figure 1: Top: Free-moving exciton (bound electron-hole pair, shown by ellipse) in a GaAs/AlGaAs quantum well. "2D exciton" means that the well width $d \ll a_{B}$, where $a_{B}$ is exciton Bohr radius in GaAs. Bottom: Schematic of the momentum conservation law $\mathbf{p}=\mathbf{k}+\mathbf{q}: \mathbf{p}$ is $2 \mathrm{D}$ exciton momentum in $\mathrm{X}-\mathrm{Y}$ plane, $\mathbf{k}$ is $3 \mathrm{D}$ photon wave vector and $\mathbf{q}=\left(\mathbf{q}_{\|}, q_{z}\right)$ is $3 \mathrm{D}$ acoustic phonon wave vector.

3D photon wave vector $\left(|\mathbf{k}|=\omega_{0} / c\right)$ and $\mathbf{q}=\left(\mathbf{q}_{\|}, q_{z}\right)$ is $3 \mathrm{D}$ acoustic phonon wave vector. By default we omit $\hbar$ in most formulae for phonon and photon momenta.

Let us suppose that a free-moving $2 \mathrm{D}$ exciton can emit a 3D photon only if $\mathbf{p}=\mathbf{k}+\mathbf{q}$, or (Fig. 1)

$$
\left(\mathbf{p}-\mathbf{q}_{\|}\right)^{2}=\mathbf{k}^{2} \sin ^{2} \theta, q_{z}=-k_{z}, \cos \theta \equiv k_{z} /|\mathbf{k}| .
$$

If $\mathbf{q}=\mathbf{0}$ the momentum conservation law (1) would allow only the formation of a $2 \mathrm{D}$ exciton-polariton in the $\mathrm{X}-\mathrm{Y}$ plane so the actual optical decay would be prohibited.

Further, let us assume that an exciton recombines instantly after scattering from an appropriate phonon (1). In particular, an exciton with initial $2 \mathrm{D}$ momentum $\mathbf{p}$ emits a $3 \mathrm{D}$ phonon with wave vector $\mathbf{q}$ so that after emission the final 2D momentum of the exciton is $\mathbf{p}-\mathbf{q}_{\|}$. The transition rate for such a process is given by

$$
w(\mathbf{p}, \mathbf{q})=\frac{2 \pi}{\hbar}\left|M_{q}\right|^{2} f_{p}\left(1+f_{p-q_{\|}}\right) \delta\left(E_{p}-E_{p-q_{\|}}-\hbar s q\right)
$$

with $q=\sqrt{q_{\|}^{2}+q_{z}^{2}}$. (Here it is implied that the excitons are bosons and the lattice temperature $T_{0}=0$.) Note that for pure 2D exciton - 3D phonon scattering, when the exciton does not decay, due to the quantum confinement the value of $q_{z}$ is undefined and one should average over it in (2).
The matrix element $M_{q}$ for the exciton-LA phonon interaction in QWs due to the deformation potential was derived in Ref.[9]. For simplicity, we consider the limiting case of zero QW width, i.e., a $2 \mathrm{D}$ layer. If $q_{\|} a_{B} \ll 1$, where $a_{B}$ is the $2 \mathrm{D}$-exciton Bohr radius, $\left|M_{q}\right|^{2}$ can be reduced to

$$
\left|M_{q}\right|^{2} \approx M^{2} a_{B}\left(q_{\|}^{2}+q_{z}^{2}\right)^{1 / 2}
$$

where $M^{2}=\frac{\hbar\left(D_{c}-D_{v}\right)^{2}}{4 \pi \rho V s a_{B}}, \rho$ is the crystal density, $V$ is its volume, $D_{c(v)}$ is the deformation potential for an electron in the conductance (valence) band, and $s$ is the sound velocity. For definiteness, in all numerical estimates we consider heavy-hole excitons in GaAs. For this material one has $a_{B} \approx 10^{-6} \mathrm{~cm}, m \approx 0.06 m_{e}\left(m_{e}\right.$ is free electron mass), $s \approx 5 \cdot 10^{5} \mathrm{~cm} / \mathrm{s}, \rho=5.3 \mathrm{~g} / \mathrm{cm}^{3}$ and $\left|D_{c}-D_{v}\right| \approx$ $10 \mathrm{eV}[3]$.

Since an exciton has a finite size and internal structure, there are two conditions required to treat the excitons as point-like structureless particles. (I) The de Broglie wavelength of an exciton should be much larger than the exciton Bohr radius, $\lambda_{d B}=\hbar / \sqrt{2 m T}>>a_{B}$. This sets the upper limit for the effective exciton temperature, $T<<E_{b}$, where $E_{b}=2 \hbar^{2} /\left(m a_{B}^{2}\right) \approx 3 \cdot 10^{2} \mathrm{~K}$ is the binding energy of a $2 \mathrm{D}$ exciton. Note that $\lambda_{d B}^{-1} \approx 10^{6}$ $\mathrm{cm}^{-1}$ at $T=10^{2} \mathrm{~K}$ and $\lambda_{d B}^{-1} \approx 3 \cdot 10^{5} \mathrm{~cm}^{-1}$ at $T=10$ $\mathrm{K}$. In addition, since we consider non-degenerate excitons, $T>T_{c}=2 \pi \hbar^{2} n / m$. Hence the theory could be applied at $1 \mathrm{~K} \lesssim T \lesssim 50 \mathrm{~K}$ with exciton density $n \lesssim 10^{10} \mathrm{~cm}^{-2}$. (II) To exclude the influence of the exciton internal structure on the exciton-acoustic phonon interaction one needs to assume that $\hbar^{2} /\left(m a_{B}^{2}\right) \gg \hbar s q$ or $q a_{B} \ll \beta^{-1}$, where $\beta=a_{B}(m s / \hbar) \approx 2 \cdot 10^{-2}$. We generalize this condition to $q a_{B} \lesssim 1$ to use both the matrix element $M_{q}$ in simplified form (3) and the fact that the photon wave vector $k \sim 10^{5} \mathrm{~cm}^{-1}$ [1] with $k a_{B} \sim 0.1$.

\section{RADIATIVE LIFETIME AND LUMINESCENCE INTENSITY}

From condition (11) it follows that for given $\mathbf{p}$ and $\mathbf{k}$ there exists only one phonon with wave vector $\mathbf{q}$ defined by (11), after the emission of which the exciton can decay radiatively. Then the average radiative lifetime $\tau_{R}$ of an exciton in the system can be determined through

$$
\frac{n}{\tau_{R}}=\frac{1}{S} \int_{0}^{\pi / 2} \frac{d \theta}{(\pi / 2)} \sum_{\mathbf{p}, \mathbf{q}_{\|}} w\left(\mathbf{p},\left(\mathbf{q}_{\|},|\mathbf{k}| \cos \theta\right)\right) \delta_{\left(\mathbf{p}-\mathbf{q}_{\|}\right)^{2}, \mathbf{k}^{2} \sin ^{2} \theta} .
$$

Here $\delta_{p, p^{\prime}}=1$ if $p=p^{\prime}$ and $\delta_{p, p^{\prime}}=0$ otherwise. On the other hand, one can determine the optical recombination rate $\Gamma(p)$ of the exciton with momentum $\mathbf{p}$ from the relation

$$
n / \tau_{R}=\frac{1}{S} \sum_{\mathbf{p}} \Gamma(p) f_{p}=I
$$




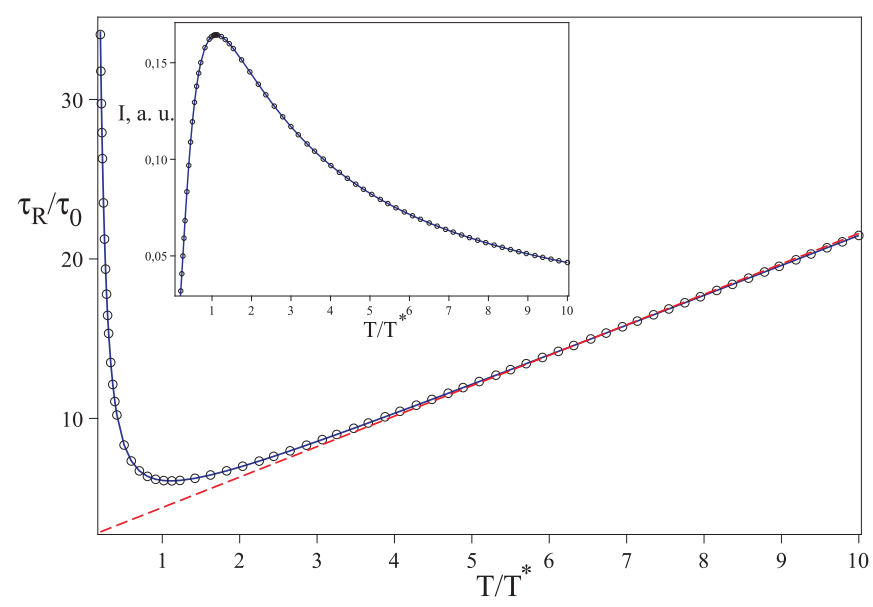

Figure 2: Radiative exciton lifetime $\tau_{R}$ as a function (9) of the effective temperature $T$ in the exciton system (lattice temperature $\left.T_{0}=0\right)$. One can see the minimum at $T=T^{*}$. Inset: luminescence intensity (7) vs $T$. Solid lines are analytical curves given by Eqs. (7), (9) with $J(x)$ in form (10), circles are numerical evaluation of Eqs. (7), (9) with $J(x)$ given by (8), and the dash line is $1.91\left(T / T^{*}\right)+2.5$.

where $I$ is the average intensity of the exciton luminescence.

Substituting (2) and (3) in Eqs. (44,5) one obtains

$\Gamma(p)=\frac{4 a_{B} M^{2}}{\hbar} \int_{0}^{\pi / 2} d \theta \sum_{\mathbf{q}_{\|}} q_{*} \delta\left(\frac{p^{2}}{2 m}-\frac{\hbar^{2} k^{2}}{2 m} \sin ^{2} \theta-\hbar s q_{*}\right)$,

with $q_{*}=\sqrt{q_{\|}^{2}+k^{2} \cos ^{2} \theta}$.

Evaluating the sums in (5) with the use of the condition $q_{\|} a_{B} \lesssim 1$, one gets (inset in Fig. 2)

$$
I=\frac{1}{S} \sum_{p} \Gamma(p) f_{p} \approx \frac{n}{\tau_{0}} x^{2} J(x)
$$

Here $x=T / T^{*}, T^{*}=\hbar s / a_{B} \approx 3.6 \mathrm{~K}, \tau_{0}^{-1}=$ $2 M^{2} S /\left(\hbar^{2} s a_{B}\right)$, where $\tau_{0}$ is the density-and-temperature independent time constant, and

$$
J(x)=\int_{0}^{\pi / 2} d \theta \exp \left(-\frac{\alpha^{2} \sin ^{2} \theta}{2 \beta x}\right) \int_{u / x}^{\frac{1}{x} \sqrt{1+u^{2}}} y^{2} d y \exp (-y)
$$

with $\alpha=k a_{B} \sim 0.1$ and $u=\alpha \cos \theta$. In the pure 2D case (i.e., for a QW with zero width) one has to consider $\tau_{0}$ as a phenomenological parameter.

According to (5), the temperature dependence of the radiative exciton lifetime is given by (Fig. 2)

$$
\tau_{R}=\tau_{0} /\left(x^{2} J(x)\right) .
$$

It is seen that at large temperature $T \gtrsim T^{*}$ the classical dependence $\tau_{R} \propto T$ is reproduced. Decreasing temperature below $T^{*}$, one finds the intuitively expected increase of $\tau_{R}$. Note that for GaAs at $T \lesssim 1 \mathrm{~K}$ the contribution of piezoelectric coupling in the exciton-acoustic phonon interaction becomes significant [10] so that one can expect smoother dependence $\tau_{R}(T)$ in this region.

Calculating the integral over $y$ in (8) and expanding $\exp \left(-\frac{\alpha}{x} \cos \theta\right)$ in the resulting expression in powers of $\alpha$ up to and including the cubic terms, then integrating over $\theta$ one gets an approximate evaluation of $J(x)$ in the form

$$
J(x) \approx F(x) G_{0}(\xi)+\frac{\alpha^{2}}{2 x^{3}} \exp (-1 / x) G_{2}(\xi)-\frac{\alpha^{3}}{3 x^{3}} G_{3}(\xi),
$$

which coincides almost exactly with the numerical solution (Fig. 2). Here $\xi=\alpha^{2} /(2 \beta x)$ and

$$
\begin{aligned}
F(x) & =2-\left(2+\frac{2}{x}+\frac{1}{x^{2}}\right) \exp (-1 / x), \\
G_{0}(x) & =\frac{\pi}{2} \exp (-x / 2) I_{0}(x / 2), \\
G_{2}(x) & =\frac{\pi}{4} \exp (-x / 2)\left(I_{0}(x / 2)+I_{1}(x / 2)\right), \\
G_{3}(x) & =\frac{1}{2 x} \exp (-x)+\frac{1}{2}\left(1-\frac{1}{2 x}\right) \sqrt{\frac{\pi}{x}} \operatorname{erf}(\sqrt{x}),
\end{aligned}
$$

where $I_{0}(x)$ and $I_{1}(x)$ are modified Bessel functions of the first kind. The approximate solution (10) allows one to obtain parameter-dependent asymptotics of (7), (9). For $x \gg 1$ one gets

$$
J(x) \approx \frac{\pi}{6 x^{3}}\left[\frac{\alpha^{6}}{(2 \beta)^{3}}+\frac{3}{4} \frac{(2 \beta)^{3}}{\alpha^{4}}-\frac{2}{3} \alpha^{3}\right] .
$$

Since $\alpha \sim \beta^{1 / 2} \sim 0.1$ one can put $J(x) \approx$ $(\pi / 6)\left(\alpha^{2} /(2 \beta)\right)^{3} / x^{3}$ when $x \gg 1$.

It is interesting to note that, according to (7) and (11), at high temperature the luminescence intensity $I \propto n / T$. The same dependence can be obtained by assuming that the intensity of the exciton luminescence is proportional to the number $N_{0}$ of excitons with zero momentum [6], $I \propto N_{0}=\exp \left(T_{c} / T\right)-1 \propto n / T$ at $T \gg T_{c}=2 \pi \hbar^{2} n / m$.

\section{CONCLUSION}

We have suggested that optical recombination of freemoving $2 \mathrm{D}$ excitons is generally induced by the excitonacoustic phonon interaction. In particular, this interaction eliminates the uncertainty of the $Z$-component of the photon momentum. The dependence of the exciton radiative lifetime $\tau_{R}$ on the effective temperature $T$ of the exciton system has been derived. At $T>T^{*} \approx 3.6 \mathrm{~K}$ the function $\tau_{R}(T)$ exhibits a well-known linear dependence. At $T=T^{*}$ the lifetime $\tau_{R}$ reaches its minimal value and at $T<T^{*}$ it increases with decreasing $T$. The characteristic temperature $T^{*}$ is determined only by the exciton Bohr radius and the sound velocity in the quantum well. For the experimental verification of the predicted nonmonotonic dependence $\tau_{R}(T)$ at low temperatures one 
needs nearly resonant exciton pumping and the lattice temperature $T_{0} \ll T^{*}$.

The author thanks L. A. Maksimov for helpful discussions, and J. Waldie, who has read the manuscript and made many useful remarks on its style.

\section{APPENDIX}

According to Refs. [4, 6] the total optical recombination rate $\Gamma(p)$ due to the $2 \mathrm{D}$ exciton - 3D photon interaction reads (as before, we omit $\hbar$ in momentum $\hbar k$, restoring the dimensionality only in the final results) $\Gamma(p)=\Gamma_{1}(p)+\Gamma_{2}(p)$,

$$
\Gamma_{1}(p)=\frac{k}{\tau_{0} \sqrt{k^{2}-p^{2}}}, \quad \Gamma_{2}(p)=\frac{\sqrt{k^{2}-p^{2}}}{\tau_{0} k},
$$

where $\mathbf{p}$ is in-plane $(\mathrm{X}-\mathrm{Y})$ momentum of a $2 \mathrm{D}$ exciton, $\mathbf{k}$ is the $3 \mathrm{D}$ photon wave vector $\left(k=\omega_{0} / c\right)$, and $\tau_{0}$ is now the radiative lifetime of an exciton with $p=0$ (note that $\tau_{0}$ in the main text has a different meaning). $\Gamma_{1}$ and $\Gamma_{2}$ correspond to different polarizations of the emitted photon.

Let us first reproduce the result of Ref.[4] by finding the average (thermal) radiative lifetime $\tau$ of an exciton if the exciton system has a Maxwell distribution function over momenta, $f_{p}=\exp \left(-\left(E_{p}-\mu\right) / T\right), E_{p}=p^{2} / 2 m$, with $|\mu| \gg T$. Then the exciton density is $n=\frac{1}{S} \sum_{\mathbf{p}} f_{p}=$ $m T \exp (\mu / T) /\left(2 \pi \hbar^{2}\right)$, where $S$ is the X-Y plane area. In the latter formula we omit the spin degeneracy factor $(=4)$ of the excitons. Defining the average intensity $I$ of the exciton luminescence as

$$
I=n / \tau=\frac{1}{S} \sum_{|\mathbf{p}|<k} \Gamma(p) f_{p}
$$

and substituting $f_{p}=\exp \left(-p^{2} /(2 m T)\right)\left(\frac{2 \pi \hbar^{2} n}{m T}\right)$ and

$$
\begin{gathered}
\sum_{|\mathbf{p}|<k}(\ldots) \approx \frac{S}{4 \pi \hbar^{2}} \int_{0}^{k^{2}} d p^{2}(\ldots), \text { one obtains } \\
I=\frac{n a}{\tau_{0}} \int_{0}^{1} d t \exp (-a t) \frac{(2-t)}{\sqrt{1-t}},
\end{gathered}
$$

with $a=k^{2} /(2 m T)$. Evaluating the integral, we finally arrive at

$$
I=\frac{n}{\tau_{0}}\left[1+\sqrt{\pi} e^{-a}\left(a-\frac{1}{2}\right) F_{1}(a)\right],
$$

where $F_{1}(a) \equiv \frac{2}{\sqrt{\pi}} \int_{0}^{1} \exp \left(a t^{2}\right) d t$.

The dependence $\tau(T)$ given by Eqs. (13) and (15) is shown in Fig. 3. At $a \ll 1$, i.e., at $T \gg k^{2} /(2 m)$ one

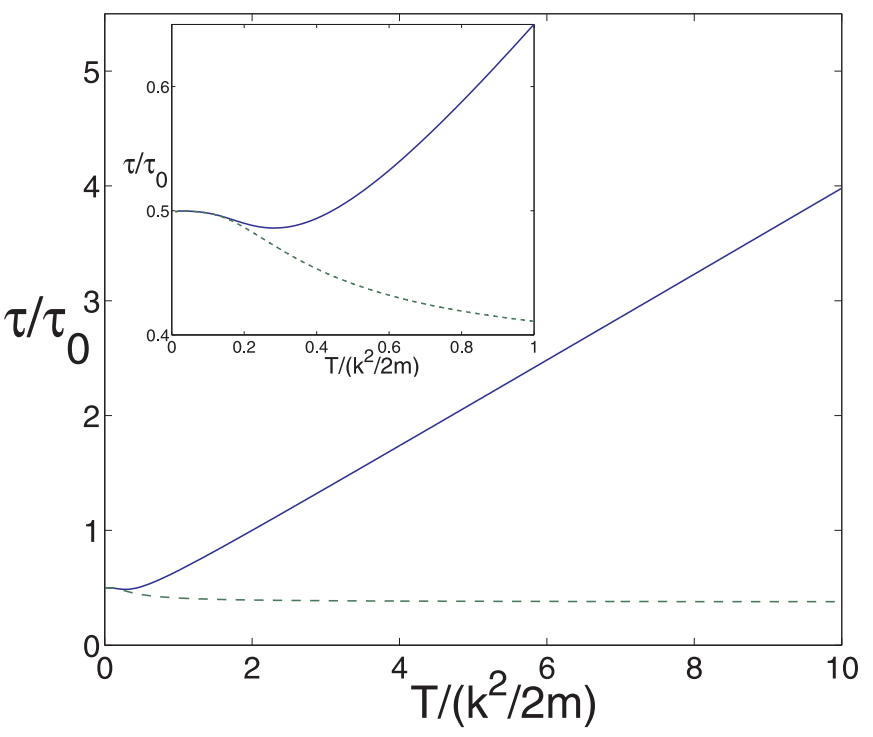

Figure 3: Radiative exciton lifetime $\tau$ given by Eqs. (13), (15) as a function of the effective exciton temperature $T$ (solid line). The dash line is $\tau(T)$ after the replacement of $n$ by $\tilde{n}$ in the left-hand side of Eq. (13). The inset is enlarged initial region of the main plot.

has $F_{1}(a) \approx 2 / \sqrt{\pi}+2 a /(3 \sqrt{\pi})$ and $I \approx \frac{8}{3} \frac{n}{\tau_{0}} a=\frac{4}{3} \frac{n}{\tau_{0}} \frac{k^{2}}{m T}$. Substituting this result in Eq.(13), we find

$$
\tau \approx \frac{3}{4} \tau_{0} \frac{m T}{\hbar^{2} k^{2}} \propto T .
$$

Formula (16) reproduces the well-known result of Ref. [4]. Note that the original result is four times larger due to the spin degeneracy factor.

At low temperature $T<\hbar^{2} k^{2} /(2 m) \sim 1 \mathrm{~K}$ the dependence $\tau(T)$ exhibits a small finite upturn (inset in Fig. 3). However, the upturn might be an artefact since, on the one hand, the temperature at the minimum is below $T_{c}$ starting with relatively low exciton densities $n \gtrsim 10^{8} \mathrm{~cm}^{-2}$ and, on the other hand, at very low density of excitons their thermalization is hindered. In Ref.[6], where the authors considered the Bose-Einstein statistics of the excitons, it was shown that the low-temperature minimum in $\tau(T)$ is absent and the curve monotonically comes to $0.5 \tau_{0}$ (in Ref.[6] it is four times larger due to the spin degeneracy factor) at $T=0$.

One should note that the definition of the average radiative lifetime of an exciton through Eq. (13) used so far may require some revision. In fact, as it is supposed, only optically active excitons (i.e., those with momenta $|\mathbf{p}|<k$ ) can contribute to the luminescence intensity. However, this has been taken into account only in the right-hand side of Eq. (13) (cp. with Eq. (5)). For consistency, one may replace the total density $n$ of excitons by the density of optically active excitons $\tilde{n}=\frac{1}{S} \sum_{|\mathbf{p}|<k} f_{p}=n(1-\exp (-a))$ in the left-hand side of Eq. (13). 
This replacement seems well-grounded when the depletion of the excitons with $|\mathbf{p}|<k$ due to their optical recombination is compensated not by the relaxation of excitons with large momenta $(|\mathbf{p}|>k)$ but by the arrival of already thermalized excitons with $|\mathbf{p}|<k$ due to the exciton pumping. Such a situation is possible with stationary resonant pumping which is balanced by the recombination.

At $a \ll 1$ one has $\tilde{n} \approx n a$. Then equating the left and the right sides in Eq. (13) one gets $\tilde{n} / \tau \approx(8 / 3)\left(n a / \tau_{0}\right)$ and $\tau \approx(3 / 8) \tau_{0}$. The next term over $a$ brings the temperature dependence (dash line in Fig. 31). Finally,

$$
\tau \approx \frac{3}{8} \tau_{0}\left(1+\frac{\hbar^{2} k^{2}}{20 m T}\right)
$$

where $T \gg \hbar^{2} k^{2} /(2 m)$. It can be seen that in the pre- vious result (16) the large factor $m T /\left(\hbar^{2} k^{2}\right) \gg 1$ appears because of the one-sided inclusion of all excitons as optically active ones, whereas at high temperatures the fraction of optically active excitons is very small $(\tilde{n} / n \sim a \ll 1)$.

The dependence $\tau(T)$ given by Eq. (17) differs qualitatively from the experimental one [8, 11], i.e., theoretical $\tau$ decreases with $T$ while in the experiments $\tau \propto T$. The discrepancy possibly comes from the pulsed pumping used in the experiments [8, 11], where the thermal relaxation timescale was much smaller than the pulse repetition period. Then the relaxation can cause a rapid re-distribution of the exciton momenta after each act of recombination and the replacement of $n$ by $\tilde{n}$ in Eq. (13) is not valid.
[1] A. L. Ivanov, L. E. Smallwood, A. T. Hammack et al., Europhys. Lett. 73, 920 (2006).

[2] V. M. Agranovich and O. A. Dubovsky, JETP Lett. 3, 223 (1966).

[3] E. Hanamura, Phys. Rev. B 38, 1228 (1988).

[4] L. C. Andreani, F. Tassone, F. Bassani, Solid State Commun. 77, 641 (1991).

[5] D. S. Citrin, Phys. Rev. B 47, 3832 (1993).

[6] A. L. Ivanov, P. B. Littlewood, H. Haug, Phys. Rev. B 59, 5032 (1999).

[7] C. Creatore, A. L. Ivanov, Phys. Rev. B 77, 075324 (2008).
[8] J. Feldmann, G. Peter, E. O. Göbel et al., Phys. Rev. Lett. 59, 2337 (1987).

[9] T. Takagahara, Phys. Rev. B 31, 6552 (1985).

[10] At fixed $\mathbf{k}$ the value of $q_{z}$ is also fixed by (1). So at large $p \sim \sqrt{2 m T}$ one has large $q_{\|}$and the main contribution is from deformation potential $\left(\left|M_{q}\right|^{2} \propto q\right)$ whereas at small $T$ the values of $q_{\|}$are also small so the main contribution can be from piezoelectric coupling (then $\left|M_{q}\right|^{2} \propto q^{-\gamma}$, $\gamma \geqslant 1)$.

[11] J. Martinez-Pastor, A. Vinattieri, L. Carraresi et al., Phys. Rev. B 47, 10456 (1993). 